-Review-

Review Series: Animal Bioresource in Japan

\title{
Current Activities of CARD as an International Core Center for Mouse Resources
}

\author{
Naomi NAKAGATA and Ken-ichi YAMAMURA \\ Center for Animal Resources and Development, Institute of Resource Development and Analysis, \\ Kumamoto University, 2-2-1 Honjo, Kumamoto 860-0811, Japan
}

\begin{abstract}
The Center for Animal Resources and Development (CARD), Institute of Resource Development and Analysis, Kumamoto University was established in 1998 based on recommendations published in the report "Preservation, Supply and Development of Genetically Engineered Animals" by the Ministry of Education, Culture, Sports, Science and Technology. We provide a comprehensive and integrated set of research services designed for the mouse-based biological research community. All services are conducted in accordance with the highest standards of animal health and genetic quality and are delivered to meet researcher's research goals. To promote biological sciences worldwide, we produce genetically engineered mice and exchangeable gene trap ES clones, cryopreserve mouse embryos and sperm, supply these resources, organize training courses to educate people, and form a hub of the domestic and international networks of both mutagenesis and resource centers. Up to now, we have produced more than 600 genetically engineered mouse strains and have more than 1,100 strains and stocks of mice for supply to the scientific community. More than 150 studies using genetically engineered mice produced or supplied by CARD have been published so far. As a founding member of the Federation of International Mouse Resources, the Asian Mouse Mutagenesis and Resource Association, and the International Gene Trap Consortium, we are contributing to the promotion of biological sciences in the world.
\end{abstract}

Key words: cryopreservation, knockout, mouse, resource, transgenic

\section{Introduction}

The Center for Animal Resources and Development (CARD), Kumamoto University was established in 1998 based on recommendations published in the report "Preservation, Supply and Development of Genetically Engineered Mice" by the Subdivision of Resource Study, Council for Science and Technology, Ministry of Educa- tion, Culture and Science on July 10, 1997. That report recommended the establishment of at least two centers which could preserve, supply, and develop genetically engineered mice in Japan. At the Institute of Molecular Embryology and Genetics, Kumamoto University, we established the Laboratory of Transgenic Technology in 1992 and started to produce transgenic mice in response to requests from scientists. This was the first trial for

(Received 26 February 2009 / Accepted 29 March 2009)

Address corresponding: K. Yamamura, Center for Animal Resources and Development, Institute of Resource Development and Analysis, Kumamoto

University, 2-2-1 Honjo, Kumamoto 860-0811, Japan 
such a service in Japan. The first transgenic mice were produced by Gordon et al. in 1980 [4]. Since then, transgenic mice have been used in many biological studies and have been shown to be a rich resource for such studies. In 1989, the first knockout mice were produced using a homologous recombination technique in embryonic stem (ES) cells [8]. With this procedure we aimed to promote scientific activities through the production of genetically engineered mice. In the mid 1990s, we realized that one transgenic mouse or knockout mouse strain can be used in many different areas of biological science, and that there was a strong demand for the preservation and supply of genetically engineered mouse strains. At the same time, the technology for the cryopreservation of mouse embryos had developed enough to be able to respond to such demand. In 2000, a new facility for embryo cryopreservation was completed and we started to cryopreserve, supply, and develop genetically engineered mice.

\section{Goals of CARD}

Our goals are as follows: 1) the establishment of mouse resources unique in Asia by integration of advanced technologies in production, cryopreservation, and the supply of genetically engineered mice; 2) the development of technologies relevant to these areas to keep our techniques advanced; 3 ) the establishment of a supply system between our center and other institutions for cryopreserved embryos or gametes to protect against infection which may arise through the transfer of live mice; 4) the training and education of workers and researchers in this field, especially those involved in reproductive engineering; and 5) the formation of domestic and international networks to promote biological sciences globally. To accomplish these goals, we became a founding member of the Federation of International Mouse Resources (FIMRe) [3]. FIMRe is a collaborating group of Mouse Repository and Resource Centers worldwide whose collective goal is to archive and provide strains of mice as cryopreserved embryos and gametes, ES cell lines, and live breeding stock to the research community. Goals of the FIMRe are (1) Coordinate repositories and resource centers to archive valuable genetically defined mice and ES cell lines being created worldwide and meet research demand for these genetically defined mice and ES cell lines, (2) Establish consistent animal health standards of the highest quality in all resource centers, (3) Provide genetic verification and quality control for genetic background and mutations, (4) Provide resource training to enhance user ability in utilizing cryopreserved resources.

In addition, we organized the Asian Mouse Mutagenesis and Resource Association (AMMRA) in 2006. The AMMRA is a collaborative group of Mouse Mutagenesis and Resource Centers in Asia. Its mission is "To promote mouse mutagenesis projects and to facilitate access to mouse resources in Asia". Its goals are "The use of mouse models for understanding genome function and the improvement of human health". The founding members of AMMRA are (1) Biological Resource Center, Singapore, (2) National Laboratory Animal Center, Taipei, (3) National Resource Center for Mutant Mice, Nanjing University, Nanjing, (4) Shanghai Institute of Biological Sciences, Shanghai, (5) Nanfang Center for Model Organisms, Shanghai, (6) Peking UniversityBLARC, Beijing, (7) Beijing Institute of Laboratory Animal Science, CAMS, PUC, Beijing, (8) Bio-Evaluation Center, KRIBB, Daejeon, (9) Riken BioResource Center, Tsukuba, (10) Center for Animal Resources and Development, Kumamoto.

\section{Characteristics of the Mouse}

Development of the laboratory mouse as a research model really began with genetic experiments in the early 1900s. Today, a large number of inbred strains of mice or various strains of mice with spontaneous or induced mutations (i.e., transgenics, targeted mutations, chemically induced mutations) have been produced in a variety of laboratories worldwide. They are prized for many qualities, including their small size, short generation time, and ease of breeding within the laboratory. The fact that they are genetically the best characterized of all mammals increases their value for all fields of study.

\section{Technical Services Available at CARD}

According to our aims, we provide a comprehensive, 
Table 1. Production, cryopreservation, supply, and cleaning of genetically engineered mice at CARD since 1998

\begin{tabular}{lcccccc}
\hline & Tg & Chimera & Total & $\begin{array}{c}\text { No. of strains } \\
\text { cryopreserved }\end{array}$ & $\begin{array}{c}\text { No. of strains } \\
\text { supplied }\end{array}$ & $\begin{array}{c}\text { No. of strains } \\
\text { cleaned }\end{array}$ \\
\hline 1998 & 67 & 6 & 73 & & & 3 \\
1999 & 22 & 1 & 23 & & & 53 \\
2000 & 66 & 11 & 77 & 144 & 4 & 62 \\
2001 & 60 & 15 & 75 & 97 & 10 & 73 \\
2002 & 59 & 21 & 80 & 67 & 34 & 47 \\
2003 & 61 & 23 & 84 & 89 & 33 & 20 \\
2004 & 44 & 14 & 58 & 116 & 58 & 65 \\
2005 & 43 & 18 & 61 & 111 & 39 & 83 \\
2006 & 23 & 13 & 36 & 153 & 42 & 83 \\
2007 & 25 & 7 & 32 & 207 & 35 & 48 \\
2008 & 37 & 6 & 43 & 142 & 53 & 106 \\
\hline Total & 507 & 135 & 642 & 1,126 & 308 & 643 \\
\hline
\end{tabular}

integrated, and highly customizable set of research services designed for the mouse-based biomedical research community. All services are conducted in accordance with the highest standards of animal health and genetic quality and are delivered to meet user's schedule and research goals. The intellectual property right of each resource belongs to the depositor, not to our center. Users should consider this issue before submitting a request for resources, and undertake signed agreements to any conditions attached to a resource.

\section{(1) Production of genetically engineered mice}

Upon requests by researchers, we produce either transgenic mice or chimeric mice. So far, we have produced 507 transgenic mouse lines and 135 chimeric mouse lines (Table 1). Researchers should prepare and send DNA constructs for microinjection into fertilized eggs. We usually use fertilized eggs obtained from C57BL/6 mice. We microinject DNA into fertilized mouse eggs and transfer them into oviducts of foster mothers. We rear new born mice up to 4 weeks of age and then send these mice to requesters. So, researchers need to carry out screening for transgenic mice at their facilities. For chimeric mouse production, researchers should prepare knockout ES clones at their facilities. We make chimeric mice by injecting targeted ES cells into blastocysts, which are then transferred to the uteri of foster mothers. We send all chimeric mice to requesters at 4 weeks of age. It should be noted that researchers should have permission for recombinant DNA experiments at their institutions.

\section{(2) Cryopreservation of embryos and sperm}

For deposit, researchers should send their mice to CARD. The numbers of mice that should be shipped to CARD for embryo freezing is as follows. For genetically engineered mouse strains, researchers should send at least 3 homozygous or 3 heterozygous male mice. Alternatively, researchers can send at least 5 homozygous males and 20 homozygous females if they desire cryopreservation of homozygous embryos. For other mouse strains, we need at least 5 males and 20 females for cryopreservation.

We generally produce embryos from these mouse strains using in vitro fertilization. Ten straws containing spermatozoa and 8 tubes containing 40 two-cell embryos are cryopreserved for each strain. We use the simple vitrification method for embryo freezing [6] and the Nakagata method for sperm freezing [5]. For quality control, some of the frozen embryos are thawed to check if the frozen embryos develop into viable young and whether or not the developed mice are microbiologically clean. For genetically engineered mouse strains, transgene transmission is confirmed by PCR analysis of genomic DNA from tail tissue of the produced mice.

Currently our center has 1,126 strains and stocks of mice. These include inbred mouse strains, spontaneous 
mutants, transgenic mice, and knockout mice. CARD R-BASE (Resource Database) provides resource-related strain, gene and reference information (http://cardb. cc.kumamoto-u.ac.jp/transgenic/index.jsp).

\section{(3) Supply of mouse strains and frozen embryos/sperm}

Cryopreserved embryos or recovered populations from cryopreserved embryos are supplied to the scientific community. The cost is $141,750 \mathrm{JY}$ or 109,200 JY for supply of recovered mice or frozen embryos, respectively. The application procedure for the supply of laboratory mice is shown on our web page (http://card medic.kumamotou.ac.jp/card/english/sigen/gyoumu/ebank/index.htm). We have already supplied 90 strains of cryopreserved embryos and 218 strains of mice worldwide.

\section{(4) Exchangeable gene trap clones (EGTC)}

Our center has more than 600 exchangeable gene trap ES clones. In general, gene trapping is a high-throughput approach that is used to introduce insertional mutations across the genome in mouse embryonic stem (ES) cells. In addition to generating standard loss-of-function alleles, newer gene trap vectors offer a variety of postinsertional modification strategies for the generation of other experimental alleles. We are using the exchangeable gene trap method for isolation of gene trap clones $[1,2,9]$. This method has a great advantage. The exchangeable gene trap vector is usually inserted around the exon containing the ATG codon in a mouse endogenous gene, resulting in the null mutation. As the $\beta$-geo gene is flanked by lox71 and loxP, the $\beta$-geo gene can be replaced with the gene of interest by electroporating a new vector containing the gene of interest flanked by lox66 and loxP together with a Cre expression vector. The newly introduced gene of interest can be expected to be expressed under the control of the trapped gene. Using this system, a mouse gene can be replaced with a homologous human gene, leading to the production of a humanized mouse at the gene level. These clones can be accessed through the EGTC database (http://egtc.jp/ view/index). As we are also the founding member of the international gene trap consortium (IGTC) [7], the EGTC database is connected to the integrated database of the IGTC. The IGTC database contains all publicly available gene trap cell lines, which are available on a non- collaborative basis for nominal handling fees. Researchers can search and browse the IGTC database for cell lines of interest using accession numbers or IDs, keywords, sequence data, tissue expression profiles, and biological pathways. At the moment, the IGTC database has 380,863 cell lines. IGTC members are BayGenomics (USA), Centre for Modelling Human Disease (Toronto, Canada), Embryonic Stem Cell Database (University of Manitoba, Canada), German Gene Trap Consortium (Germany), Sanger Institute Gene Trap Resource (Cambridge, UK), Soriano Lab Gene Trap Database (Mount Sinai School of Medicine, New York, USA), Texas Institute for Genomic Medicine-TIGM (USA), TIGEM-IRBM Gene Trap (Naples, Italy), and our institution, Exchangeable Gene Trap Clones (Kumamoto University, Japan).

\section{(5) Other resources}

In the Gene Technology Center, which also belongs to the Institute of Resource Development and Analysis, we started the GTC P-stock service in April 2004. This is not a plasmid bank; however, we do store valuable transgenes or targeting constructs for homologous recombination and supply them upon request (http://gtc. egtc.jp/view/pstock/index).

\section{(6) Courses and education}

CARD sponsors stimulating courses and conferences on our campus at Honjo in Kumamoto and at other locations inside and outside Japan. CARD training courses for reproductive engineering techniques were introduced in November 2000. Spring and autumn courses are staged each year at CARD. The courses teach the following techniques:1) Preparing and Assembling Pipettes for Embryo Handling, 2) In Vitro Fertilization, 3) Simple Vitrification of Mouse Embryos, 4) Vitrification and Transplantation of Mouse Ovaries, 5) Cryopreservation of Mouse Spermatozoa, 6) In Vitro Fertilization using Cryopreserved Spermatozoa, 7) Collecting Two-CellStage Embryos, 8) Vasectomy for the Creation of Sterile Males, 9) Embryo Transfer into the Oviduct, 10) Production of Chimeric Mice by 8-Cell Aggregation, 11) Embryo Transfer into the Uterus, and 12) Caesarean Section and Fostering. We sometimes hold the training course at other venues inside and outside Japan. 


\section{Popular Strains: Characteristic Features and Their Use for Research}

Table 2 shows the mouse strains which are requested by many researchers. A recent trend is that requests for Cre mice are increasing substantially due to the performance of its conditional knockout.

Among Cre mice, P0-Cre is one of the most frequently requested strains. Although this strain of mouse has been described in a published manuscript [10], we would like to briefly introduce the characteristics of this mouse. Neural crest cells are embryonic, multipotent stem cells that give rise to various cell/tissue types and thus serve as a good model system for the study of cell specification and mechanisms of cell differentiation. For analysis of neural crest cell lineage, transgenic mice harboring a Cre gene driven by a promoter of protein 0 (P0) were generated. To detect and visualize the Cre-mediated DNA recombination in neural crest cells and derivatives, we utilized another transgenic (Tg) line with the CAG-CAT$\mathrm{Z}$ indicator construct [10]. This $\mathrm{Tg}$ line carries a $l a c \mathrm{Z}$ reporter gene downstream of a chicken $\beta$-actin promoter and a "stuffer" fragment flanked by two loxP sequences. The lacZ is expressed only when the stuffer is removed by the action of Cre recombinase. Using these transgenic mice, we demonstrated that a subset of migrating neural crest cells and a wide variety of cells in the neural crest cell lineage could be marked by lacZ expression. Thus, the use of this system may facilitate many interesting experiments, including lineage analysis, purification, and manipulation of the mammalian neural crest cells. Also, this cell-type-specific transgenesis system should facilitate functional analysis of genes of interest in the neural crest cell lineage. Up to now, this strain of mouse has been used in many studies and is now one of the most useful Cre-driver mice.

\section{Deposition and Request for Mouse Strains and EGTC}

An outline of the deposition and request procedures for mouse strains is illustrated in Fig. 1. Information on the application procedure is available on the web page (Fig. 2) (http://card.medic.kumamoto-u.ac.jp/card/english/index.html). As we are one of the founding members
Table 2. Frequently requested mouse strains

\begin{tabular}{|c|c|}
\hline ID: 148 & C57BL/6J-Tg(PO-Cre)94Imeg \\
\hline ID: 250 & $\operatorname{Tg}(\mathrm{K} 5$-Cre $)$ \\
\hline ID: 709 & B6;C3H-Tg(K19-Wnt1/K19-Ptgs2/K19-Ptges) \\
\hline ID: 428 & C57BL/6N-Tg(CAG-AURKA(WT)Card \\
\hline ID: 196 & B6;129-Synd $4^{\text {tm } 1}$ \\
\hline ID: 189 & $\operatorname{Tg}($ E/nestin:EGFP-50) \\
\hline ID: 175 & $\operatorname{Tg}(\mathrm{E} /$ nestin:EGFP-25) \\
\hline ID: 91 & $\mathrm{Tg}(\mathrm{CAG}-\mathrm{Cre})$ \\
\hline ID: 89 & $\operatorname{Tg}($ lck-Cre $)$ \\
\hline ID: 509 & B6;D2-Tg(CAG-CAT-EGFP)39Miya \\
\hline ID: 355 & C57BL/6-CD9 $9^{\mathrm{tm} 1} ; \operatorname{Tg}(\mathrm{ZP} 3-\mathrm{EGFPCD} 9)$ \\
\hline ID: 312 & B6;CB-Dtr ${ }^{\operatorname{tm} 2(l o x)}$ \\
\hline ID: 290 & C57BL/6 -Tg(Act-EGFP)C14-Y01-FM131Osb \\
\hline ID: 88 & $\mathrm{C} 57 \mathrm{BL} / 6-\operatorname{Rag} 1^{\mathrm{tm} 1(\mathrm{GFP}) I m k u}$ \\
\hline
\end{tabular}

of FIMRe (http://www.fimre.org/), we also offer our mouse data to the integrated database IMSR (International Mouse Strain Resources) (http://www.informatics. jax.org/imsr/index.jsp). Thus, researchers also can find mouse strains using these databases.

\section{Major Achievement: Publication}

More than 150 studies using genetically engineered mice produced by CARD, have been published so far. Among these some representative manuscripts are listed in Table 3. This list clearly suggests that technical services in mouse embryo manipulation and reproductive engineering contribute greatly to the promotion of science.

\section{Related Information}

JMSR (http://www.shigen.nig.ac.jp/mouse/jmsr/top . jsp): The Japan Mouse/Rat Strain Resources Database (JMSR) is a searchable online database of mouse/rat strains and stocks available in Japan. The JMSR was developed in 2001 through discussions with the Mouse Genetic Resources Subcommittee (http://www.shigen. nig.ac.jp/shigen/grc/grc_mouse.jsp). The objective of this database is to provide a portal site which will help users to locate and obtain mouse/rat resources. This site is maintained by the Genetic Informatics Laboratory, National Institute of Genetics in close cooperation with data providers.

IGTC (http://www.genetrap.org/): The International 


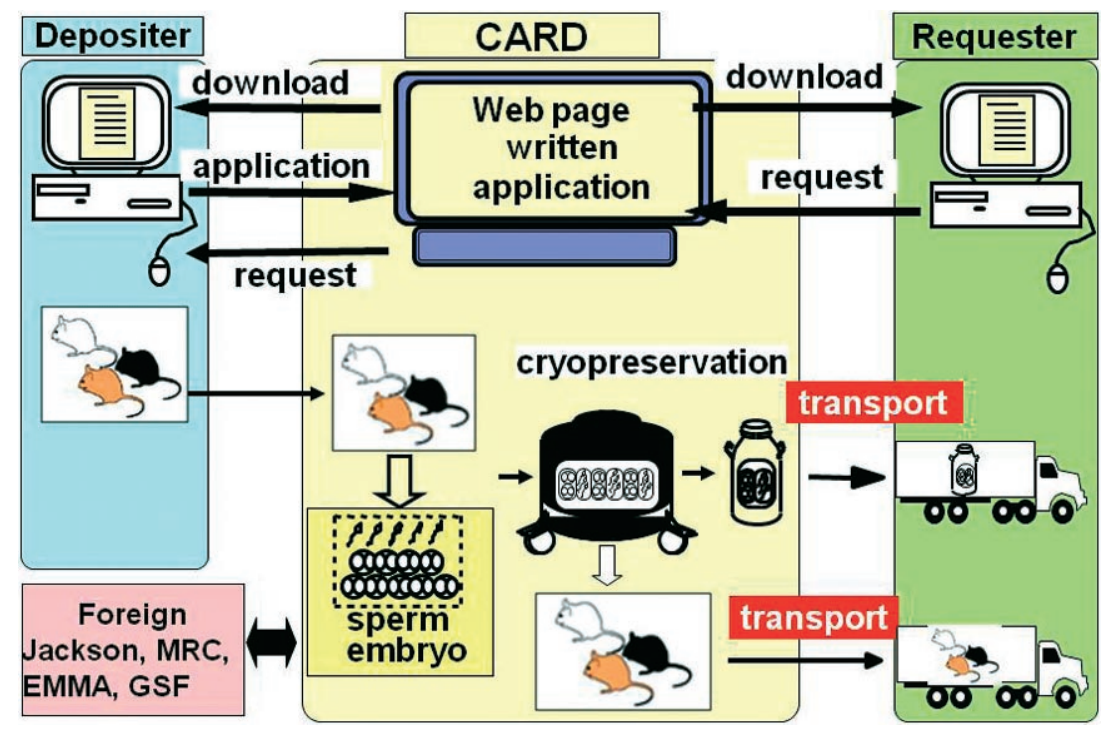

Fig. 1. Outline of deposition and request procedures for mouse strains. Information on the application procedure and necessary documents are available on the web page. Mouse embryos or sperm obtained from mice sent from depositors are cryopreserved and stored in liquid nitrogen tanks. Frozen embryos or live mice obtained by transfer of thawed embryos into foster mothers can be sent to requesters.

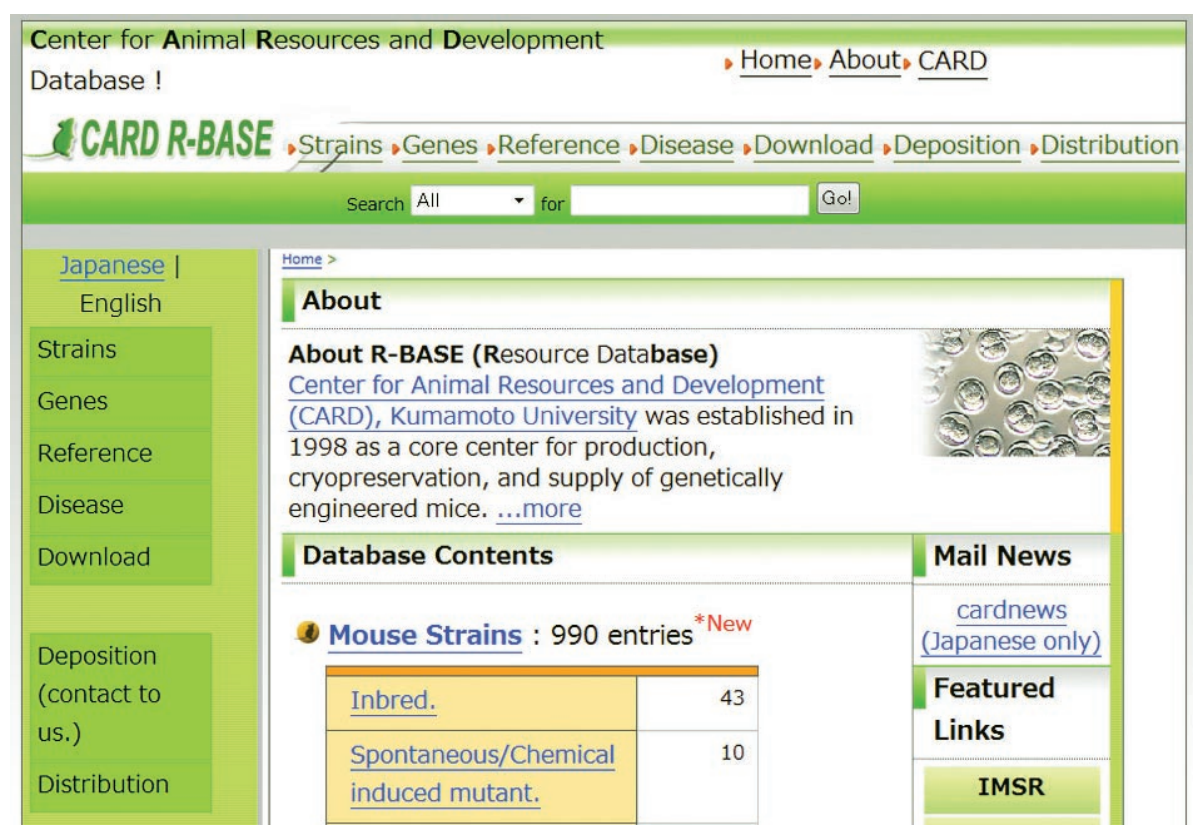

Fig. 2. Web page of CARD R-BASE. Only part of the web page is shown in this figure. Both Japanese and English versions are available.

Gene Trap Consortium (IGTC) consists of laboratories around the world working together to generate a public library of mutated murine ES cell lines. Such cell lines can be obtained on a non-collaborative basis by scientists interested in generating reporter-tagged, loss-of-function mutations in mice. In addition to loss of function, new- 
Table 3. Selected publications

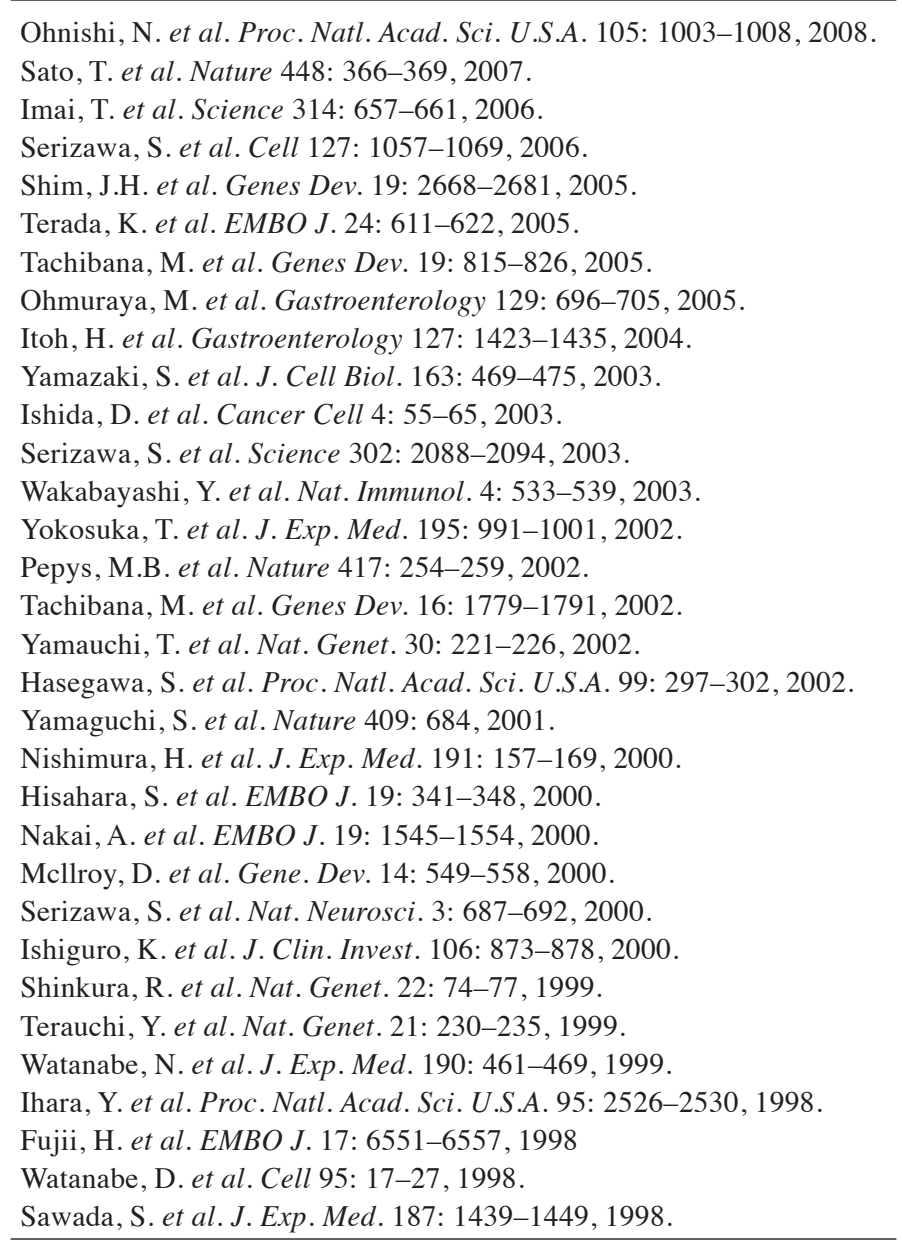

er gene trap vectors offer a variety of post-insertional modification strategies to allow for the generation of other experimental alleles. The cooperative goal of the IGTC is to generate an international resource representing all or most genes in the mouse genome, and to provide the bioinformatics and logistical support to make the resource valuable and available to scientists.

IMSR (http://www.informatics.jax.org/imsr/index.jsp): The International Mouse Strain Resources (IMSR) is a searchable online database of mouse strains and stocks available worldwide, including inbred, mutant, and genetically engineered mice. The goal of the IMSR is to assist the international scientific community in locating and obtaining mouse resources for research. The data content found in the IMSR is as it was supplied by data provider sites.

EUCOMM (http://www.eucomm.org/): The European
Conditional Mouse Mutagenesis Integrated Project (EUCOMM) is funded by the European Union Framework 6 programme. The goal of EUCOMM is to generate a collection of up to 13,000 mutated genes in mouse C57BL/6N embryonic stem (ES) cells using conditional gene trapping and gene targeting approaches. This library will enable mouse mutants to be established worldwide in a standardized and cost-effective manner, making mouse mutants available to a much wider biomedical research community than has been possible previously.

NorCOMM (http://norcomm.phenogenomics.ca/): NorCOMM (North American Conditional Mouse Mutagenesis project) is a large-scale research initiative focused on developing and distributing a library of mouse embryonic stem (ES) cell lines carrying single conditional knockout mutations across the mouse genome. 
KOMP (http://www.nih.gov/science/models/mousel knockout/): The Knockout Mouse Project is a trans-NIH initiative that aims to generate a comprehensive and public resource comprised of mouse embryonic stem (ES) cells containing a null mutation in every gene in the mouse genome.

\section{References}

1. Araki, K., Araki, M., and Yamamura, K. 2002. Site-directed integration of the cre gene mediated by Cre recombinase using a combination of mutant lox sites. Nucleic Acids Res. 30: e103.

2. Araki, K., Imaizumi, T., Sekimoto, T., Yoshinobu, K., Yoshimuta, J., Akizuki, M., Miura, K., Araki, M., and Yamamura, K. 1999. Exchangeable gene trap using the Cre/ mutated lox system. Cell. Mol. Biol. (Noisy-le-grand) 45: $737-750$.

3. Davisson, M. 2006. FIMRe: Federation of International Mouse Resources: global networking of resource centers. Mamm. Genome 17: 363-364.

4. Gordon, J.W., Scangos, G.A., Plotkin, D.J., Barbosa, J.A., and Ruddle, F.H. 1980. Genetic transformation of mouse embryos by microinjection of purified DNA. Proc. Natl. Acad.Sci. U.S.A. 77: 7380-7384.

5. Nakagata, N. 2000. Cryopreservation of mouse spermatozoa.
Mamm. Genome 11: 572-576.

6. Nakao, K., Nakagata, N., and Katsuki, M. 1997. Simple and efficient vitrification procedure for cryopreservation of mouse embryos. Exp. Anim. 46: 231-234.

7. Nord, A.S., Chang, P.J., Conklin, B.R., Cox, A.V., Harper, C.A., Hicks, G.G., Huang, C.C., Johns, S.J., Kawamoto, M., Liu, S., Meng, E.C., Morris, J.H., Rossant, J., Ruiz, P., Skarnes, W.C., Soriano, P., Stanford, W.L., Stryke, D., von Melchner, H., Wurst, W., Yamamura, K., Young, S.G., Babbitt, P.C., and Ferrin, T.E. 2006. The International Gene Trap Consortium Website: a portal to all publicly available gene trap cell lines in mouse. Nucleic Acids Res. 34: D642-648.

8. Schwartzberg, P.L., Goff, S.P., and Robertson, E.J. 1989. Germ-line transmission of a c-abl mutation produced by targeted gene disruption in ES cells. Science 246: 799803.

9. Taniwaki, T., Haruna, K., Nakamura, H., Sekimoto, T., Oike, Y., Imaizumi, T., Saito, F., Muta, M., Soejima, Y., Utoh, A., Nakagata, N., Araki, M., Yamamura, K., and Araki, K. 2005. Characterization of an exchangeable gene trap using pU-17 carrying a stop codon-beta geo cassette. Dev. Growth Differ. 47: 163-172.

10. Yamauchi, Y., Abe, K., Mantani, A., Hitoshi, Y., Suzuki, M., Osuzu, F., Kuratani, S., and Yamamura, K.1999. A novel transgenic technique that allows specific marking of the neural crest cell lineage in mice. Dev. Biol. 212: 191-203. 